\title{
Reducing Cost Through Standardization
}

\author{
Kevin G. Friedman, $M D^{1,2}$ \\ David R. Fulton, $M D^{1,2, *}$
}

\author{
Address \\ ${ }^{1}$ Harvard Medical School, Boston, MA, USA \\ ${ }^{*}, 2$ Department of Cardiology, Boston Children's Hospital, 300 Longwood Avenue, \\ Boston, MA, 02115, USA \\ Email: david.fulton@cardio.chboston.org
}

Published online: 19 October 2016

(C) Springer International Publishing AG 2016

This article is part of the Topical Collection on Quality Improvement

Keywords Standardization - Cost · Guidelines - Quality · SCAMPs · Algorithms

\section{Opinion statement}

The drive for standardization of care within pediatrics based on medical evidence is gaining momentum among hospitals and providers. Initiated by care guidelines with origins in specialty societies, adoption of pathways is now being implemented in many settings. The keys to success of these algorithms in narrowing practice variation and their potential contribution to reducing utilization of unnecessary resources are not uniformly addressed in advance. Clinical guidelines are often not updated frequently to incorporate new evidence from the literature. Education of providers about the goals and process of pathways in advance may not be sufficient depending on the commitment of the clinical champions of the algorithms. Anticipatory modeling to identify potential barriers in the workflow that interfere with proper implementation of pathways is beneficial with the opportunity to refine the algorithm where appropriate. In order to confirm cost reduction, economic data points need to be defined in advance for comparisons between preimplementation and post-implementation periods. It is possible that improved outcomes from narrowed practice variation may not always lead to cost reduction which should be reconciled by analysis of the data. Our experience with these limitations motivated our Department of Cardiology to seek a different approach to care pathways, standardized clinical and management pathways (SCAMPs) which engender algorithm design informed by existing evidence and experience of clinicians coupled with targeted questions that can be addressed by active data collection. Providers are free to depart from recommendations in the algorithm but must explain their reasons, an option that promotes participation. Data analysis combined with new medical evidence leads to iterative changes, greater standardization, and reduction of cost. 


\section{Introduction}

Escalating costs in medicine are a gnawing threat to the national economic prosperity. The theme is not new, but serious efforts to address the expense of medical practice have emerged only in the past decade. The most compelling influence has been the call from the Institute of Medicine to improve medical care by concentrating on improvement in six major domains: safety, efficacy, patient-centered care, time, equity, and efficiency [1]. The implication of these changes is that health care delivery will result in better outcomes for patients and that changes in the focus of physicians will necessarily lead to more sensible use of resources through standardized approaches to diagnostics and management. As Berwick has pointed out, the triple aim of the health care system should be improving the individual experience, improving health overall, and reducing cost of providing care [2]. In the absence of prior attention to these goals, health care spending has continued to escalate increasing by $5.3 \%$ in 2014 to approximately $\$ 3.0$ trillion or $\$ 9523$ per capita equivalent to $17.5 \%$ of gross domestic product (https:// www.cms.gov/research-statistics-data-and-systems/statistics-trends-and-reports/nationalhealthexpenddata/ nationalhealthaccountshistorical.html). There is little disagreement argument that this trajectory is unsustainable. The intent of this review is to identify recent examples of care standardization that have shown effectiveness in reducing medical costs. In addition, we will highlight a novel approach to quality improvement that has been used in our hospital, other institutions as well as specialty provider groups that address reduction of unnecessary utilization of resources as a key driver.
From an industry perspective, medicine evolved for a long period of time without concern for its underlying costs. The incentives inherent in the system functioned in contrast to control of expenses. Physicians were paid and still are to varying degrees, on a fee-for-service basis that sets no limits to diagnostic evaluation and therapeutic management. The freedom to consider and order any serologic or imaging test was left solely at the discretion of the practitioner regardless of the lack of evidence in the literature to support the efficacy of the given diagnostic approach. Furthermore, there was no restriction on the frequency with which these tests could be ordered without a defined understanding of whether a given test would provide more insight into the nature of the cause of the patient's condition or its treatment. Both hospitals and physicians were aligned in this effort since reimbursement associated with the care was financially rewarded.

However, as third party payers began to impose some limits to reimbursement, hospital revenue streams came under pressure. The budgetary reality meant that hospitals could achieve a balance in the ideal setting by reducing those expenditures not likely to contribute to improved care. However, variation in the care provided by the physicians remained a driving force in determining the ultimate expenditures for a given patient in terms of diagnostics and therapeutics. To achieve reduction in variability of practice with its anticipated downstream impact of decreasing unnecessary testing, physicians needed to manage conditions by using a framework of logical steps, though limited by the lack of substantial medical evidence.

\section{Standardization}

As more evidence appeared in the literature to support specific diagnostic and therapeutic options, specialty societies began to establish clinical guidelines that might be applied to manage a specific condition [3-9]. Creation of such pathways was the result of the amalgamation of medical evidence gleaned from the literature and the combined experience of experts tasked with writing these care plans. Once adopted, however, the pathways can remain stagnant without alteration depending upon how frequently the champions of such guidelines revise them. In the absence of updated care plans, new evidence that could contribute to improved outcomes or reduction in utilization of unnecessary resources into an existing guideline may be unknown to providers. Other limitations to consistent implementation of such guidelines are the variability 


\section{Cost}

in patient conditions necessitating alterations in the diagnostics or therapeutics recommended by the algorithm. A fierce sense of independence on the part of physicians also counteracts the well-intentioned concept of uniformity of action implied in guidelines. Furthermore, it is difficult to measure how often guidelines are followed unless mandated locally or used as a metric in pay for performance by insurance plans.

On a wide scale, it is virtually not possible to know how costs may have been reduced as a result of such guidelines. On the other hand, some pediatric hospital systems have committed to broad implementation of care plans in the daily workflow [10-13]. Many clinical practice guidelines address single disease states or conditions within pediatric networks, single hospitals, or other user groups. The focus can relate to a diagnostic strategy, therapeutic intervention, or change in process. Successful implementation and function of care algorithms is dependent upon creation and ongoing support of the infrastructure needed to address the elements of the care plan. Perhaps the most important ingredient is the commitment and enthusiasm of the leaders of a given care plan who assemble a team to create and evaluate the plan based on available knowledge. Once completed, user education is essential prior to initiation of the plan, and trial modeling can identify barriers to utilization in the workflow. In the case of assessing the impact of the algorithm on cost, analyzing resource utilization prior to standardization is mandatory for comparison once the pathway is active.

Though the overall goal of those in the health care field should be to reduce unnecessary costs, the pediatric share of expenditures in medicine is relatively small. Nonetheless, improving quality of care should always have the intent of optimizing expenditures to drive value. The determination of cost can be extensive in its components. Single episodes of hospital-based care in its most simplistic fashion can be measured by the direct cost of its component parts including personnel, equipment, ancillaries, and overhead composing a daily rate as well as supplies, diagnostic testing, therapy all composing the hospital costs. Additionally, physician billing incorporates the remaining portion of expenditures.

Though health care economists prefer to perform cost utility analysis to determine the impact of illness on the allocation of scarce resources $[14,15]$, given longevity and developmental changes over the first two decades as well as suboptimal methodologic tools for the age ranges in question, such estimates are not frequently addressed in pediatrics [1618 ], referenced in only $8 \%$ of pediatric studies through 2012 [14]. One other point to consider is that cost effectiveness analysis compares two different health plans or treatments with regard to outcomes and cost. A plan that results in no change in outcome with reduced cost or improved outcomes at no additional cost would favor use of that approach. However, in this methodology, cost reduction can drive standardization, while the object of this review is to assess the impact of standardization on cost. 
A final factor to consider is the additional cost of supporting the algorithm borne by either the hospital and/or physicians. In the model used at Seattle Children's Hospital in 2010 [19], standardized clinical pathways were designed for 15 different conditions which were evidenced based to the degree possible, embedded in the order sets to promote adherence and tracked for outcomes. Using an interrupted time series over a 4-year period with a 1 -year look back at the preimplementation phase, the overall costs decreased by $\$ 155 /$ month, with a slope of -0.03 days/admission/month without an effect on patient physical functioning or readmissions. Of interest, with respect to the individual pathways, only two were associated with a statistically significant cost reduction while five others showed a trend in reduction that did not meet significance attributed to the low volume of patients treated using the pathways. Physician adherence to the pathways was not measured, so it is possible that greater cost reductions might have been achieved with improved participation. This success attained with these cost reductions however must be balanced against the programmatic costs of $\$ 17$ million dollars over 5 years, a figure that would be prohibitive for most other groups to duplicate.

\section{Standardization in networks}

The potential for greatest cost reduction are standardized models implemented across a network of health care providers. In a report of a care pathway for asthma undertaken at eight hospitals in the pediatric hospital network of Intermountain Healthcare, readmissions and length of stay at the tertiary care hospital decreased with a trend toward significant cost reduction which persisted over a 5 -year period. For the community hospitals, however, the readmission rate did not change significantly, but length of stay and cost both decreased though the sample size was small at the community hospitals [13]. In another report from a multicenter study involving 21 hospitals [19], providers used a consensus-derived pathway for inpatients with bronchiolitis and showed reduction in resource utilization from pre-implementation measurements including use of any bronchodilator (29\%), use of any steroid (68\%), chest radiography (44\%), and length of stay $(5 \mathrm{~h})$. Though cost reduction can probably be assumed, no specific cost data was analyzed. In another study, a clinical pathway for asthma used in a tertiary pediatric hospital was introduced into a pediatric unit of an adult community hospital with standardized order sets. Cost data identified a pre-post reduction in costs from $\$ 2010$ to $\$ 1174$ per patient [20]. Further evidence to support cost reduction through standardization across multiple sites was reported based on use of the Low Risk Ankle Rule, an algorithm shown to reduce radiographs for children without fractures or clinically unimportant ankle fractures [21]. The rule was implemented in three Canadian emergency departments with costs compared to three sites not using the rule to assess possible fractures. The number of important fractures missed was negligible for patients assessed with the rule as opposed to the non-rule group. Overall, the costs associated with use of the rule decreased by $\$ 36.93$ per patient, mostly 
attributable to decreased cost of radiographs and follow-up surgical visits though the cost for implementation was $\$ 6.28$ per patient [22].

\section{Standardization in hospitals}

Some pediatric hospitals have committed to standardization by introducing widespread implementation of care pathways. Rady Children's Hospital has been using clinical practice guidelines throughout the institution for nearly two decades with reduction of costs [11]. Antimicrobial stewardship programs are specific guidelines for the use of antibiotics sanctioned by the Infectious Diseases Society of America and the Society for Healthcare Epidemiology of America [23]. The primary objective is to improve clinical outcomes while reducing toxicity and organism resistance while simultaneously reducing cost. Agwu et al. initiated a web-based antimicrobial stewardship program in place of a prior restricted process requiring direct communication with infectious disease specialists [24]. It achieved a cost savings of $\$ 370,069$ for restricted agents without a change in the cost for unrestricted antimicrobials. In a follow-up paper, Sick et al. showed that cost reduction was sustained over a 5-year period by using the electronic algorithm with an annual savings of only $\$ 104,000$ before factoring the yearly cost for maintaining the program, a difference modulated by the fact that the emergency department and ICU were permitted to use a first dose of restricted drugs [25]. Newland and colleagues used a program where feedback was provided prospectively to clinicians on selected antibiotics though use was not restricted. Physician compliance with the recommendations was $92 \%$ [26]. Though cost was not analyzed, antibiotic usage for total days of select drugs decreased pre-to-post stewardship by $6 \%$ monthly for all antibiotic usage and $12 \%$ monthly for select antibiotics.

\section{Standardization for specific conditions}

Opportunities for cost reduction in specific conditions have been identified in various individual settings. Using the framework for standardization of treatment of bronchiolitis [4], Akenroye implemented an algorithm to reduce unnecessary utilization of resources in the emergency department (ED) using it to evaluate 2929 patients with the clinical diagnosis of bronchiolitis over more than six seasons [27]. Total costs included those incurred in the ED and inpatient costs if patients were not discharged from the ED. Compared with preimplementation practice, by using an uninterrupted time series, cost reductions were found in the use of chest radiographs $(-21 \%)$, RSV testing $(-11 \%)$, and use of albuterol $(-6 \%)$. The total mean cost per patient was reduced $\$ 197$ with a total cost savings of $\$ 196,409$ over 2 years.

Using a guideline for evaluation of patients up to 21 years in the ED with isolated skull fractures diagnosed by either CT scan or skull radiograph, Lyons et al. recommended discharge without hospitalization for those with normal mental status, non-focal neurologic findings, and no concern for non-accidental trauma followed by telephone assessment within 2 weeks [28]. Though cost data was not analyzed, the admission rate for such patients was reduced from $71 \%$ pre-implementation to $24 \%$ post-implementation over a 7 -year period. No patient was identified who required readmission to the hospital. 
Syncope is a frequent chief complaint in pediatric emergency departments, though generally a benign condition without an underlying cardiac or neurologic etiology. Guse et al. designed an evidence-based guideline with the primary intent of reducing testing in the ED [29]. The only recommended testing for patients considered at low risk was an electrocardiogram and pregnancy testing for post-menarche females. Compared to the pre-implementation period, testing under the guidelines decreased for CBC (36 to $16 \%$ ), dextrose sticks (33 to $15 \%$ ), and serum electrolytes ( 29 to $12 \%$ ). Virtually all patients were discharged to home. By phone follow-up in $69 \%$ of the population, visits to specialists occurred in $13 \%$ (cardiology) and $7 \%$ (neurology) with only $10 \%$ of these patients undergoing subsequent testing. Cost data were not analyzed though by inference, costs were decreased without a change in outcomes.

Cost reduction following a change in the type of catheter used in ventricular shunts placed for hydrocephalus was analyzed by Attenello et al. [30]. In changing from standard catheters to antibiotic-impregnated shunts to reduce shunt infections, 13 patients $(3.2 \%)$ with the antibiotic-impregnated shunts developed infections compared with 25 patients (12\%). This difference resulted in a cost differential of $\$ 1,234,928$ for the 18 months of standard catheter usage as opposed to $\$ 606,328$ over 4 years for the impregnated catheters, a reduction entirely related to the decreased number of infections encountered with the impregnated catheters.

\section{Standardization of process}

The role of standardization through changes in process can play a valuable role in cost reduction without changing the management itself. Baker utilized a standardized approach to the discharge of ventilator-dependent patients by creating pathways to facilitate team teaching and communication leading to discharge [31]. Compared with a pre-intervention group, the process improvement reduced length of stay in the respiratory care unit by $56 \%$ and by $42 \%$ for overall hospital length of stay. The direct hospital non-physician costs were reduced by $43 \%$ though other simultaneous improvements may have contributed to the savings. Lindgren addressed cost reduction for behavioral therapy for children with autism by comparing three different approaches for behavioral modification including in-home therapy, home telehealth, and clinic-based telehealth. The treatment outcomes did not vary, but total facility, provider, and family costs were reduced by a minimum of $49 \%$ per treatment period. Another opportunity to reduce costs is the use of checklists and decision support to limit the utilization of unnecessary laboratory testing [32]. Algaze and others demonstrated marked reduction in a number of basic laboratory tests by using these methods in a cardiac intensive care unit that resulted in actual yearly cost savings of $\$ 717,538$ without negative impact on clinical outcomes or mortality rate or length of stay [33].

\section{Standardized clinical and management pathways}

Based on the most recent evidence and experience, clinical pathway guidelines have led to improved outcomes and in some cases to cost reduction of the episode of care. Overall, such guidelines act to raise physician performance to a 
higher level, but further enhancements require updating algorithms based on new evidence derived from the literature. The contribution of outcomes from randomized controlled trials is potentially beneficial, but such evidence may not be timely, applicable to the patient cohort in question, expensive, or not actively addressed at all. Though the assumption is that costs decrease once the CPGs are adopted, the efforts to measure the financial impact are not often in place. To address these multiple issues, we have developed a quality improvement tool known as standardized assessment and management plans (SCAMPs) which has been in place for more than 7 years.

In response to the changing landscape of medicine described above, we developed a novel quality improvement tool that aims to reduce practice variation and optimize resource utilization, while also improving patient care. We termed this initiative SCAMPs. SCAMPs are an innovative and systematic approach to gathering and acting on relevant clinical data through care pathways. The process of SCAMP design and implementation has been previously described in detail [34-36]. Initially implemented in pediatric cardiology and subsequently throughout our Children's Hospital and in several adult and pediatric hospitals across the country and internationally, SCAMPs had its roots in attempts to standardize care through the development of clinical practice guidelines. The paucity of definitive medical evidence and data-driven standard of care for many conditions in many domains of medicine, as well as the recognition that most clinical practice guidelines have a limited life span, hindered our ability to develop and maintain useful clinical practice guidelines. Each SCAMP is developed by a multidisciplinary committee of physician and nursing experts for a particular medical condition, who, after a thorough review of the literature, construct an assessment and management algorithm through a consensus-based process. Similar to a CPG, a SCAMP provides a guideline that standardizes the assessment and management of patients with a specific disorder through use of care delivery algorithms. One of the underlying hypotheses of this initiative is that standardizing care will lead to reduced practice variation and optimization of resource utilization which will in most, but not all, cases lead to a reduction in resource utilization by eliminating unnecessary testing.

SCAMPs have several unique features that distinguish them from other quality improvement methodologies that aim to reduce practice variation. The impetus for the creation of SCAMPs was in part related to a lack of evidence-based guidelines and difficulty in conducting large-scale, prospective, randomized clinical trials within many fields of medicine, which ideally form the basis of algorithms for clinical practice guidelines. Lacking randomized controlled trials for many pediatric conditions, SCAMPs have been developed to address conditions where there is no definitive evidence to establish best practice but only sound practice based upon existing data in combination with expert clinical consensus. In contrast to clinical practice guidelines which are often static, the SCAMP process includes rigorous data collection. Systematic data collection and the assumption that the SCAMP care pathway can be improved allow for information feedback to facilitate iterative modification of the care delivery algorithm based on the data collected. SCAMPs also differ from clinical practice guidelines in that there is a built-in mechanism for monitoring physician adherence and an expectation for knowledge-based deviations from care pathway. Uniquely, SCAMP acknowledges uncertainty inherent in its management recommendations and therefore invites and collects 
data on knowledge and experience-based clinician deviations from the care pathway [37].

To date, SCAMPs have been implemented at nine hospitals and via two regional/national medical societies. There are currently 37 active pediatric SCAMPs at Boston Children's Hospital and our pediatric partners with over 45,000 patients enrolled (Fig. 1 and Table 1). Regional implementation of pediatric SCAMPs for evaluation of chest pain and syncope in children and adolescents has been successful and well-received by both providers and patients $[38,39]$. The first hurdle facing any care pathway is achieving physician buy-in, which can be partially assessed by measuring adherence and resultant practice variation. The quality improvement literature has consistently shown that reducing practice variation leads to better patient outcomes, decreased patient care costs, and improved efficiency. Adherence to SCAMPs has varied based on complexity of the SCAMP and diversity of the patient population but has generally been $\sim 80 \%$ (Fig. 2). Despite the fact that SCAMPs encourage knowledge-based deviations, this adherence rate exceeds that of most clinical practice guidelines.

Evaluation of the effect of SCAMP implementation on practice variation, cost of care, and quality of care is ongoing. When properly conceived and implemented, SCAMPs can reduce practice variation, which has led to a reduction in resource utilization. The pediatric chest pain SCAMP has been the most thoroughly evaluated SCAMP to date. Although chest pain is a common presenting compliant, the overwhelming majority of chest pain in otherwise healthy children has a non-cardiac etiology [34-37]. Despite the low prevalence of serious cardiac pathology in children with chest pain, extensive and costly cardiac evaluation is common. To reduce variability in cardiac testing and thereby optimize resource utilization while maintaining or improving quality of care, we developed an algorithm, which forms the basis of the chest pain SCAMP using history, physical exam, and electrocardiogram to suggest when further testing. Generally, an echocardiogram is indicated [40]. This algorithm is targeted at identifying relevant cardiac causes of chest pain while also reducing practice variation by eliminating low-yield testing, including exercise stress tests and outpatient rhythm monitors in patients without palpitations.

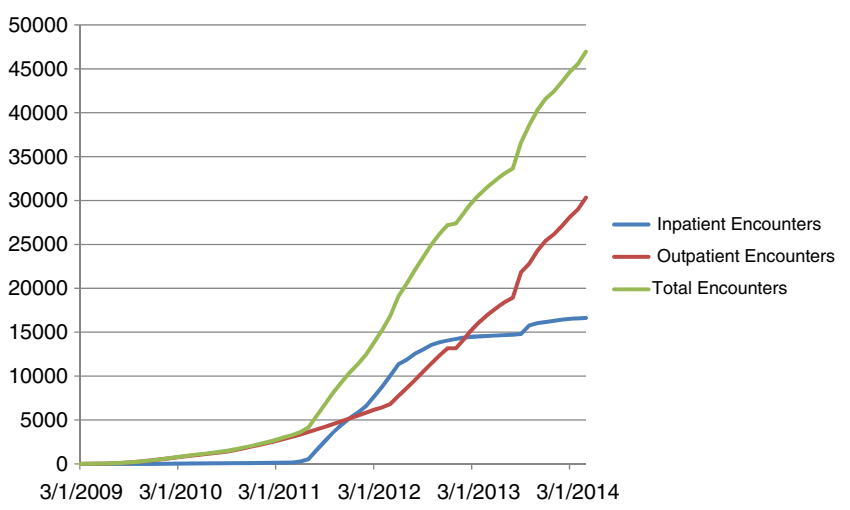

Fig. 1. Total patient encounters managed using SCAMPs at Boston Children's Hospital. *Adapted from Farias et al. Health Affairs. 2013;32:911-20. 


\section{Table 1. Active pediatric SCAMPs}

-Airway disorders

-Aortic regurgitation

-Aortic stenosis

-Aortic stenosis catheterization lab management

-Arterial switch operation

-Blood ordering/cell salvage

-Chest pain

- Coarctation

- Cognitive and headache management

- Critical asthma

- Cytomegalovirus prevention

-Dilated aorta

-Distal radius fracture

- ECMO anticoagulation

-Food challenge

-Hypertrophic cardiomyopathy

-Hyperparathyroidism

-Immune thrombocytopenia

-Interstage single ventricle

- Lipid management

- Lipid PCP

- Myocarditis

- Neonatal patent ductus arteriosus

-Nutrition

- Operative management of tetralogy of fallot

-Orthodontic retention

-PICC line placement

-Polycystic ovarian syndrome

-PPHN

- Sedation and analgesia for ventilated pts

- Skin abscess

- Small patent ductus arteriosus

-Somatoform disorders

-Syncope

-Ureterocele

-Wolff-Parkinson-White syndrome 


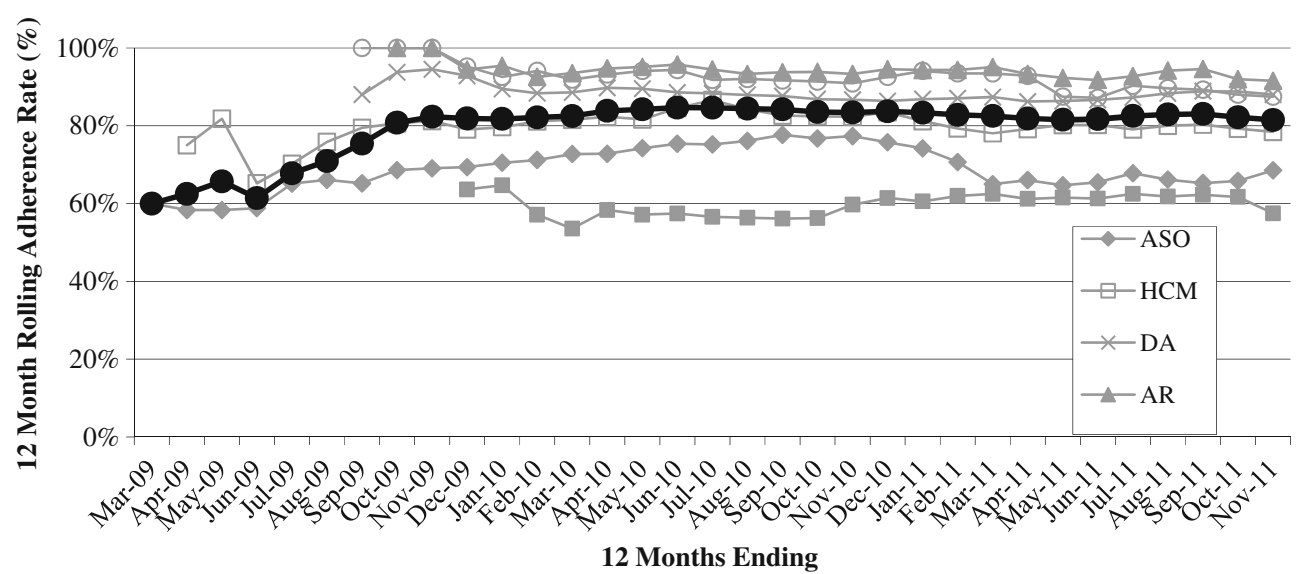

Fig. 2. Twelve-month rolling scamp adherence rate for the initial six SCAMPs. ASO arterial switch operation, HCM hypertrophic cardiomyopathy, DA dilated aorta, AR aortic regurgitation, $A S$ aortic stenosis, SV single ventricle. *Adapted from Farias et al. Congenital Heart Disease 2011;6:558-65.

We analyzed a historical cohort of pediatric chest pain patients seen in the year preceding SCAMP implementation and predicted that the utilization of several diagnostic tests including echocardiograms, exercise stress tests (EST), and outpatient rhythm monitors could be reduced by $\sim 20 \%$ or more using the chest pain SCAMP without negatively affecting patient care [40]. After 2 years of SCAMP implementation, we compared practice variation and resource utilization in cardiac testing in the pre-SCAMP cohort to the patients managed using the SCAMP [41]. Provider adherence to the chest pain SCAMP was $\sim 84 \%$, and this contributed to a significant decrease in practice variation. Of 16 possible testing patterns (combinations of four most commonly used cardiac tests), 13 were observed in the historical cohort compared to eight in the SCAMP cohort. This represented a statistically significant reduction in practice variation ( $p$ $<0.001$ ). Resource utilization also significantly decreased for a number of diagnostic testing modalities commonly used in the evaluation of chest pain. In the pre-SCAMP cohort, nearly $30 \%$ of patients underwent exercise stress testing, despite a lack of established clinical utility demonstrated in several studies [38-41]. In the SCAMP period, utilization of this test decreased to $\sim 3 \%$ of patients. Similarly, outpatient rhythm monitor usage was reduced from $\sim 10$ to $\sim 2.5 \%$ of patients with SCAMP implementation. The estimated cost savings was $\$ 206,863$ over a 1 -year study period. Importantly, no abnormalities were detected on cardiac evaluation that represented a cardiac etiology of chest pain.

Table 2. Resource utilization and cost of care after implementation of six SCAMPs

\begin{tabular}{lllllll}
\hline & Chest pain & ASO & HCM & AS clinic & AR & AS, cath lab 10-year total \\
Number of SCAMP pts & 399 & 188 & 226 & 83 & 111 & 14 \\
Control costs/patient episode & $\$ 1506$ & 2384 & 1638 & 5406 & 2064 & 34,100 \\
SCAMP costs/patient episode & $\$ 1200$ & 2111 & 1306 & 2661 & 1464 & 22,743 \\
$\%$ Reduction & $20 \%$ & $11 \%$ & $20 \%$ & $51 \%$ & $30 \%$ & $33 \%$
\end{tabular}




\section{A. Provider Survey}

How positive or negative is your opinion of

SCAMPs?

Which evidencebased method do you prefer?
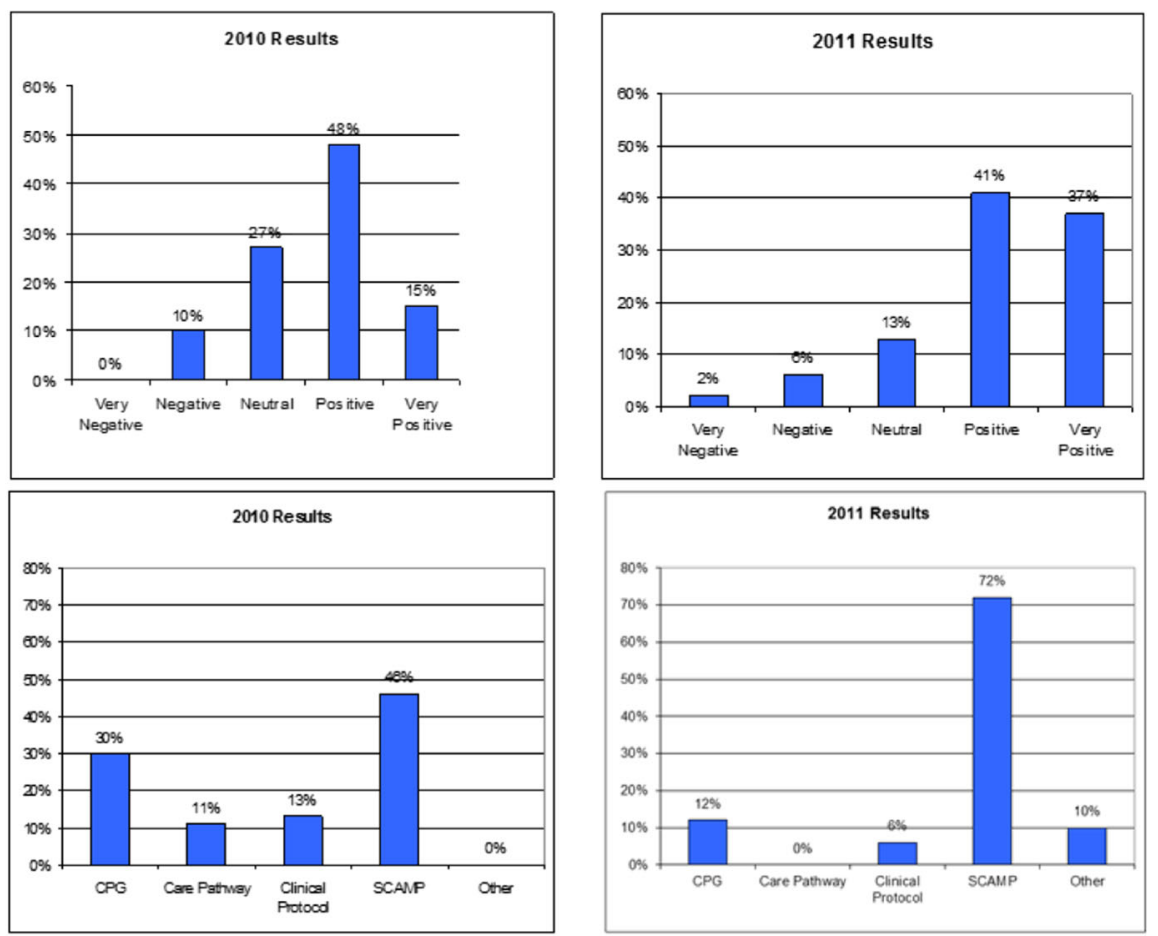

\section{B. Patient Survey}

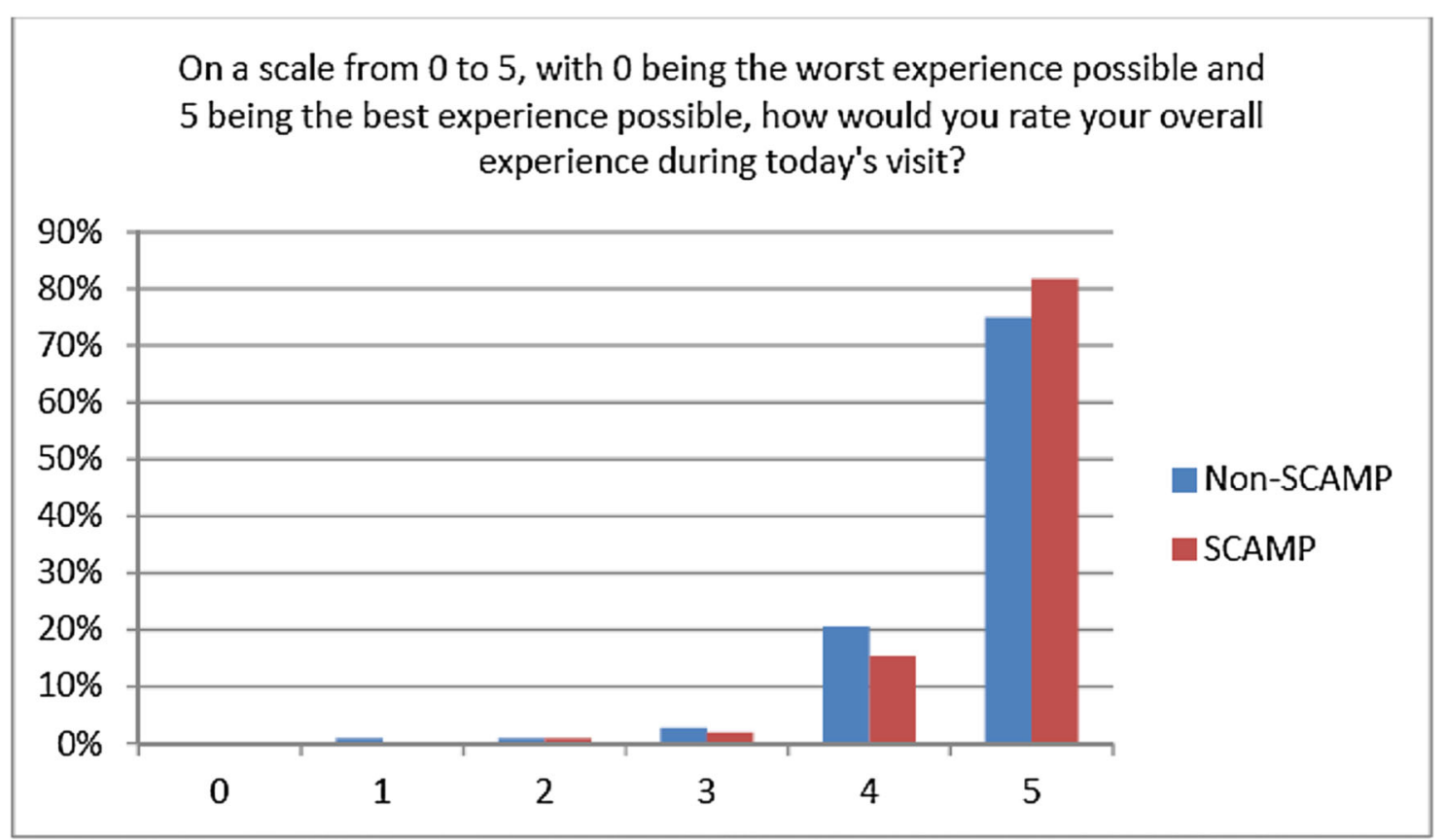

Fig. 3. Results of provider and patient experience survey. a Provider survey results* $\mathbf{b}$ Patient survey results. *Adapted from Farias et al. Congenital Heart Disease 2011;6:558-65. 
SCAMPs addressing a wide range of pediatric and adult conditions have shown promising initial results for reduction in practice variation. Examples of SCAMPs decreasing practice variation and unnecessary resource utilization include adult and pediatric conditions as well as SCAMP targeting procedures. The initial six pediatric cardiology SCAMPs were reviewed after 2 years of SCAMP implementation and resource utilization, and costs were compared to a historical control cohort from the year prior to SCAMP implementation (unpublished data, Tables 1 and 2). Implementation of all six of these SCAMPs resulted in a reduction in patient care costs with decreases ranging from 11 to $51 \%$ of costs.

In the Boston Children's' Department of Orthopedic Surgery, wide practice variation in treatment of distal radius torus fractures and the lack of utility of a routine 4-week X-ray for distal radius fracture follow-up was noted. A SCAMP for evaluation and management of pediatric distal radius fracture was developed and implemented with a resultant cost savings of $14 \%$ [42].

A SCAMP targeting optimization of testing patterns for confirmation of food allergies has been evaluated. One of the goals of the SCAMP was to reduce the number of patients undergoing allergy testing in a high-resource infusion center instead of in the clinic. SCAMP implementation resulted in an increase in the number of children who completed food challenges successfully and a significant decrease in the proportion of challenges ordered in the higher resource location [43].

Lastly, a SCAMP aiming to standardize the approach to percutaneous dilation of stenotic aortic valves in children showed reduction in practice variation with all patients managed by using the SCAMP achieving the acute procedural goal (reduction in aortic stenosis to mild or less) compared to $\sim 60 \%$ of the historical control group [44]. Improved acute outcomes attributable to standardization are predicted to lead to fewer repeat interventions to address late valve dysfunction, but this will require validation over time $[44,45]$.

Importantly, the reductions in practice variation and resource utilization associated with SCAMP implementation have not resulted in a reduction in patient or provider satisfaction. In fact, patient and provider satisfaction was similar or better by using SCAMPs. Farias et al. published a survey of comparing provider's opinions on SCAMPs before and after SCAMP implementation [46] and showed that most providers had a positive or very positive opinion of SCAMPs, and compared to other quality improvement tools, SCAMPs were the preferred methodology (Fig. 3a). A patient experience survey was administered after outpatient clinic visits to 100 patients with conditions managed by using a SCAMP and 100 control patients (unpublished data, Fig. 3b). Both groups were blinded to whether or not the clinic visit involved use of a SCAMP. Results showed a trend toward higher patient satisfaction in visits that were managed by using a SCAMP.

\section{Conclusion}

The emphasis on quality improvement in medicine is facilitated by reduced variation in practice by using evidence-based guidelines. Providers have an obligation to minimize unnecessary utilization of resources which by extension places limitations on costs. However, it is important to challenge guidelines as less than final products by recognizing the continuing contribution of published data in order to improve outcomes. As part of the equation, however, 
providers should keep high on their radar screens the costs attached to medical decision making. New quality improvement tools such as SCAMPs can play an important role in addressing management of common and rare conditions, especially since randomized controlled trials are expensive, time-intensive, and answer a select number of questions. Rapid cycle, iterative changes in algorithms based on analysis of collected data, can foster improved outcomes, lower costs, and increase provider satisfaction.

\section{Acknowledgments}

Supported in part by the Tommy Kaplan Family Fund.

\section{Compliance with Ethical Standards}

\section{Conflict of Interest}

Kevin G. Friedman declares that she has no conflict of interest.

David R. Fulton reports personal fees from UpToDate.

Human and Animal Rights and Informed Consent

This article does not contain any studies with human or animal subjects performed by any of the authors.

\section{References and Recommended Reading}

Papers of particular interest, published recently, have been

highlighted as:

- Of importance

1. Institute of Medicine, Committee on Quality Health Care in America. Crossing the quality chasm: a new health system for the 21st century. Washington: National Academies Press; 2001.

2. Berwick DM, Nolan TW, Whittington J. The triple aim: care, health and cost. Health Aff (Millwood).

2008;27(3):759-69.

3. Floto RA, Olivier KN, Saiman L, US Cystic Fibrosis Foundation and European Cystic Fibrosis Society, et al. US cystic fibrosis foundation and European cystic fibrosis society consensus recommendations for the management of non-tuberculous mycobacteria in individuals with cystic fibrosis. Thorax. 2016;71 Suppl $1: 1-22$.

4. American Academy of Pediatrics Subcommittee on Diagnosis Management of Bronchiolitis. Diagnosis and management of bronchiolitis. Pediatrics. 2006;118:1774-93.

5. Committee on Infectious Diseases. From the American Academy of Pediatrics: policy statements-modified recommendations for use of palivizumab for prevention of respiratory syncytial virus infections. Pediatrics. 2009;124(6):1694-701.

6. Vandenplas Y, Rudolph CD, Di Lorenzo C, North American Society for Pediatric Gastroenterology Hepatology and Nutrition, European Society for Pediatric Gastroenterology Hepatology and Nutrition, et al. Pediatric gastroesophageal reflux clinical practice guidelines: joint recommendations of the north American society for pediatric gastroenterology, hepatology, and nutrition (NASPGHAN) and the European society for pediatric gastroenterology, hepatology, and nutrition (ESPGHAN). J Pediatr Gastroenterol Nutr. 2009;49(4):498-547.

7. Kemper AR, Mahle WT, Martin GR, et al. Strategies for implementing screening for critical congenital heart disease. Pediatrics. 2011;128(5):e1259-67.

8. Monagle P, Chan AKC, Neil A, Goldenberg NA, et al. Antithrombotic therapy in neonates and children: antithrombotic therapy and prevention of thrombosis, 
9th ed: American college of chest physicians evidencebased clinical practice guidelines. Chest. 2012;141(2 Suppl):e737S-801.

9. Thornton PS, Stanley CA, De Leon DD, et al. Recommendations from the pediatric endocrine society for evaluation and management of persistent hypoglycemia in neonates, infants, and children. J Pediatr. 2015;167(2):238-45.

10. Mandel KE, Muething SE, Schhoetker PJ, et al. Transforming safety and effectiveness in pediatric hospital care locally and nationally. Pediatr Clin N Am. 2009;56:905-18.

11. Kurtin P, Stucky E. Standardize to excellence: improving the quality and safety of care with clinical pathways. Pediatr Clin N Am. 2009;56:893-904.

12. Lion KC, Wright DR, Spencer S, et al. Standardized clinical pathways for hospitalized children and outcomes. Pediatrics. 2016;137(4):e20151202. This report is important since it describes the successful implementation of multiple standardized pathways in a single institution with evidence of cost reduction from the pre-implementation period.

13. Nkoy F, Fassi B, Stone B, et al. Improving pediatric asthma care and outcomes across multiple hospitals. Pediatrics. 2015;136(6):e1602.

14. Kromm SK, Bethell J, Kraglund F, et al. Characteristics and quality of pediatric cost-utility analyses. Qual Life Res. 2012;21:1315-25.

15. Drummond MFSM, Torrand GW, O'Brien BJ, et al. Methods for the economic evaluation of healthcare programs. 3rd ed. New York: Oxford University Press; 2005.

16. Petrou $\mathrm{S}$. Methodological issues raised by preferencebased approaches to measuring the health status of children. Health Econ. 2003;12:697-702.

17. Griebsch I, Coast J, Brown J. Quality adjusted life years lack quality in pediatric care: a critical review of published cost utility studies in child health. Pediatrics. 2005; 115:e600-14.

18. Tarride JE, Burke N, Bischof $\mathrm{M}$, et al. A review of health utilities across conditions common in paediatric and adult populations. Health Qual Life Outcomes. 2010;8:12.

19. Ralston SL, Garber MD, Rice-Conboy E, et al. A multicenter collaborative to reduce unnecessary care in inpatient bronchiolitis. Pediatrics. 2016;137.

20. Dayal A, Alvarez F. The effect of implementation of standardized, evidence-based order sets on the efficiency and quality measures for pediatric respiratory illnesses in a community hospital. Hospital Pediatrics. 2015;5:624-9.

21. Boutis K, Grootendorst P, Willan A. Effect of the low risk ankle rule on the frequency of radiography in children with ankle injuries. CMAJ. 2013;185:731-8.

22. Boutis $\mathrm{K}$, von Keyserlingk $\mathrm{C}$, Willan A. Cost consequence analysis of implementing the low risk ankle rule in emergency departments. Ann Emerg Med. 2015;66(5):455-63.

23. Dellit T, Owens RC, McGowan JE, et al. Infectious diseases society of America and the society for healthcare epidemiology of America guidelines for developing an institutional program to enhance antimicrobial stewardship. Clin Infect Dis. 2007;44:159-77.

24. Agwu AL, Lee CK, Jain SK. A world wide web-based antimicrobial stewardship program improves efficiency, communication, and user satisfaction and reduces cost in a tertiary care pediatric medical center. Clin Infect Dis. 2008;47(6):747-53.

25. Sick AC, Lehmann CU, Tamma PD, et al. Sustained savings from a longitudinal cost analysis of an internetbased preapproval antimicrobial stewardship program. Infect Control Hosp Epidemiol. 2013;34(6):573-80. The study demonstrates that the stewardship program introduced to narrow practice variation among providers has had long term efficacy.

26. Newland JG, Stach LM, De Lurgio AS, et al. Impact of a prospective-audit-with-feedback antimicrobial stewardship program at a children's hospital. Jour Pediatr Infect Dis. 2012;1:179-86.

27. Akenroye AT, Baskin MN, Samnaliev M, et al. Impact of a bronchiolitis guideline on ED resource use and cost: a segmented time series analysis. Pediatrics.

2014; 133:e227-34.

28. Lyons TW, Stack AM, Monuteaux MC, et al. A QI initiative to reduce hospitalization with isolated skull fractures. Pediatrics. 2016;137(6):e20153370.

29. Guse SE, Neuman MI, O'Brien M, et al. Implementing a guideline to improve management of syncope in the emergency department. Pediatrics. 2014;134:e1413-21. Attenello FJ, Garces-Ambrossi GL, Zaidi HA, et al. Hospital costs associated with shunt infections in patients receiving antibiotic-impregnated shunt catheters versus standard shunt catheters. Neurosurgery. 2010;66(2):284-9.

31. Baker CD, Martin S, Thrasher J et al. A standardized discharge process decreases length of stay for ventilatordependent children. Pediatrics. 2016 137(4). Pii: e20150637.

This study shows the contribution of standardization of process to cost reduction without changing management of the underlying condition.

32. Lindgren S, Wacker D, Suess A, et al. Telehealth and autism: treating challenging behavior at lower cost. Pediatrics. 2016;137 Suppl 2:S167-75.

33. Algaze CA, Wood M, Pageler NM et al. Use of a checklist and clinical decision support tool reduces laboratory use and improves cost. Pediatrics. 2016; 137(1).

34. Rathod RH, Farias M, Friedman KG, et al. A novel approach to gathering and acting on relevant clinical information: SCAMPS. Congenit Heart Dis. 2010;5:543-53.

35. Friedman KG, Rathod RH, Farias M, et al. Resource utilization after introduction of standardized clinical assessment and management plan. Congenit Heart Dis. 2010;5:374-81.

36. Farias M, Jenkins K, Lock J, et al. Standardized clinical assessment and management plans (SCAMPS) provide a better alternative to clinical practice guidelines. Health Aff. 2013;32:911-20. 
37. Farias M, Friedman KG, Powell AJ, et al. Dynamic evolution of practice guidelines: analysis of deviations from assessment and management plans. Pediatrics. 2012;130:93-8.

38. Angoff GH, Kane DA, Giddins N, et al. Regional implementation of pediatric cardiology chest pain guideline using SCAMPS methodology. Pediatrics. 2013;132:E1010-7.

39. Paris Y, Toro-Salazar OH, Gauthier NS, et al. Regional implementation of a pediatric cardiology syncope algorithm using standardized clinical assessment and management plans (SCAMPS) methodology. J Am Heart Assoc. 2016;5. The report is an example of the ability to scale a pathway among different sized and types of groups acting collaboratively within a network.

40. Friedman KG, Kane DA, Rathod RH, et al. Management of pediatric chest pain using a standardized assessment and management plan. Pediatrics. 2011;128:239-45.

41. Verghese GR, Friedman KG, Rathod RH, et al. Resource utilization reduction for the evaluation of chest pain in pediatrics using a novel standardized clinical assessment and management plan (SCAMP). J Am Heart Assoc. 2012;1.
42. Waters PM. Value in pediatric orthopedic surgery health care: the role of time-driven activity-based cost accounting (TDABC) and standardized clinical assessment and management plans (SCAMPs). J Pediatr Orthop. 2015;35:S45-7. This paper presents a rigorous cost accounting methodology to determine the value of a SCAMP to manage distal radius fracture.

43. Simberloff T, Parambi R, Bartnikas LM, et al. Implementation of a standardized clinical assessment and management plan (SCAMP) for food challenges. J Allergy Clin Immunol Pract. 2016.

44. Porras D, Brown DW, Rathod RH, et al. Acute outcomes after introduction of a standardized clinical assessment and management plan (SCAMP) for balloon aortic valvuloplasty in congenital aortic stenosis. Congenit Heart Dis. 2014;9:316-25.

45. Brown DW, Dipilato AE, Chong EC, Lock JE, McElhinney DB. Aortic valve reinterventions after balloon aortic valvuloplasty for congenital aortic stenosis intermediate and late follow-up. J Am Coll Cardiol. 2010;56:1740-9.

46. Farias M, Ziniel S, Rathod RH, et al. Provider attitudes toward standardized clinical assessment and management plans (SCAMPs). Congenit Heart Dis.

2011;6:558-65. 